\title{
Large area, continuous, few-layered graphene as anodes in organic photovoltaic devices
}

Cite as: Appl. Phys. Lett. 95, 063302 (2009); https://doi.org/10.1063/1.3204698

Submitted: 19 May 2009 . Accepted: 23 July 2009 . Published Online: 13 August 2009

Yu Wang, Xiaohong Chen, Yulin Zhong, Furong Zhu, and Kian Ping Loh

\section{ARTICLES YOU MAY BE INTERESTED IN}

Organic solar cells with solution-processed graphene transparent electrodes Applied Physics Letters 92, 263302 (2008); https://doi.org/10.1063/1.2924771

Organic solar cells with carbon nanotube network electrodes

Applied Physics Letters 88, 233506 (2006); https://doi.org/10.1063/1.2209887

Production of a 100-m-long high-quality graphene transparent conductive film by roll-toroll chemical vapor deposition and transfer process

Applied Physics Letters 102, 023112 (2013); https://doi.org/10.1063/1.4776707

Challenge us.

What are your needs for periodic signal detection?

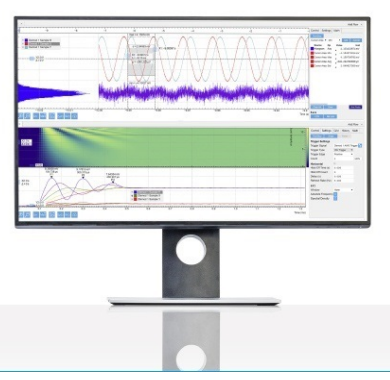

- Zurich

- Instruments 


\title{
Large area, continuous, few-layered graphene as anodes in organic photovoltaic devices
}

\author{
Yu Wang, ${ }^{1}$ Xiaohong Chen, ${ }^{1}$ Yulin Zhong, ${ }^{1}$ Furong $Z \mathrm{Zhu}^{2}$ and Kian Ping Loh ${ }^{1, \mathrm{a})}$ \\ ${ }^{1}$ Department of Chemistry, National University of Singapore, 3 Science Drive 3, Singapore 117543, Singapore \\ ${ }^{2}$ Institute of Materials Research and Engineering, 3 Research Link, Singapore 117602, Singapore
}

(Received 19 May 2009; accepted 23 July 2009; published online 13 August 2009)

\begin{abstract}
Large-area, continuous, transparent, and highly conducting few-layered graphene films produced by chemical vapor deposition method were used as anode for application in photovoltaic devices. The noncovalent modification of the graphene films with pyrene buanoic acid succidymidyl ester improved the power conversion efficiency (PCE) to $1.71 \%$. This performance corresponds to $\sim 55.2 \%$ of the PCE of a control device based on indium tin oxide (ITO)/poly $(3,4-$ ethylenedioxythiophene):poly(styrenesulfonate)/poly(3-hexyl)thiophene: phenyl- $\mathrm{C}_{61}$-butyric acid methyl ester/LiF/Al electrodes $(\mathrm{PCE}=3.1 \%)$. This finding paves the way for the substitution of ITO in photovoltaic and electroluminescent devices with low cost graphene films. (C) 2009 American Institute of Physics. [DOI: 10.1063/1.3204698]
\end{abstract}

Graphene has been extensively studied due to its unique electronic and mechanical properties as well as its eagerly projected role in the all-carbon post complementary metaloxide-semiconductor technological revolution. ${ }^{1-3}$ Its twodimensional (2D) aromatic sheet structure, as well as its high conductivity, transparency, mechanical strength, and flexibility, impart great advantages on graphene as a candidate material for the development of "plastic electronics." $"$ " The flexibility of graphene contrasts with the fragility of indium tin oxide (ITO). In principle, cost should not be a major issue for the production of graphene since its precursor is hydrocarbon gas. This is advantageous for the bulk production of electrodes when compared to the high cost of ITO electrodes and the depleting supply of indium in the world. Several studies have investigated the potential of graphene as transparent electrodes in organic electronics using chemically exfoliated graphene oxides, which were thermally reduced. However, the power conversion efficiency (PCE) of these chemically prepared graphene when deployed as anode in dye sensitized solid state electrode was found to be only $0.26 \% .^{9}$ The group of Wu et al. ${ }^{10}$ showed a marginally improved PCE of $0.4 \%$ using $\mathrm{CuPc}$ and $\mathrm{C}_{60}$ as the photoactive materials. For chemically processed graphene, the performance is limited mainly by the contact resistance of the small graphene flakes and the insulating property of graphene chemically reduced from graphene oxide. The structural defects and lateral disorder of such chemically exfoliated graphite affects the carrier mobility of the film negatively. ${ }^{11}$ Therefore, although graphene could provide a low cost alternative to ITO, there is still tremendous room for improvement in terms of enlarging the lateral scale of graphene in these devices.

In the present study, we synthesized large-area graphene film on Ni film-coated $\mathrm{SiO}_{3} / \mathrm{Si}$ wafer using chemical vapor deposition (CVD) process. ${ }^{12}$ To transfer the graphene films, the wet etching of Ni film was first carried out using $\mathrm{FeCl}_{3}$ and $\mathrm{HCl}$ solution. Subsequent to that, dry-transfer technology based on polydimethylsiloxane (PDMS) stamp was used to other substrate directly. ${ }^{12}$ Figure 1 (a) shows typical scanning electron microscopy (SEM) image of the graphene film

${ }^{a)}$ Electronic mail: chmlohkp@nus.edu.sg. on Si substrate, where it can be seen that the entire graphene film is continuous on a wide lateral scale. The thickness of graphene film on the $\mathrm{SiO}_{2} / \mathrm{Si}$ substrate can be distinguished by optical microscopy because of the strong amplitude modulation of reflection at the air-grapheme- $\mathrm{SiO}_{2}$ interface [the inset of Fig. 1(a)]. ${ }^{13}$ High-resolution transmission electron microscopy (HRTEM) image in Fig. 1(b) shows a wrinkled paperlike structure at low magnification. In the inset of Fig. 1(b), selected area electron diffraction indicates several sets of strong diffraction spots distributed on polycrystalline rings. The incommensurate stacking between the layers is responsible for the Moiré pattern. ${ }^{14}$ Moreover, according to the profile and position of the Raman 2D band as well as the ratio of the intensities of the $\mathrm{G}$ and $2 \mathrm{D}$ band, it can be concluded that the graphene film contain micrometer sized domains corresponding to one, two, and multilayered (greater than three layers) graphene [Fig. 1(c)]. ${ }^{15}$ There is no appreciable D-band around $1350 \mathrm{~cm}^{-1}$ associated with de-
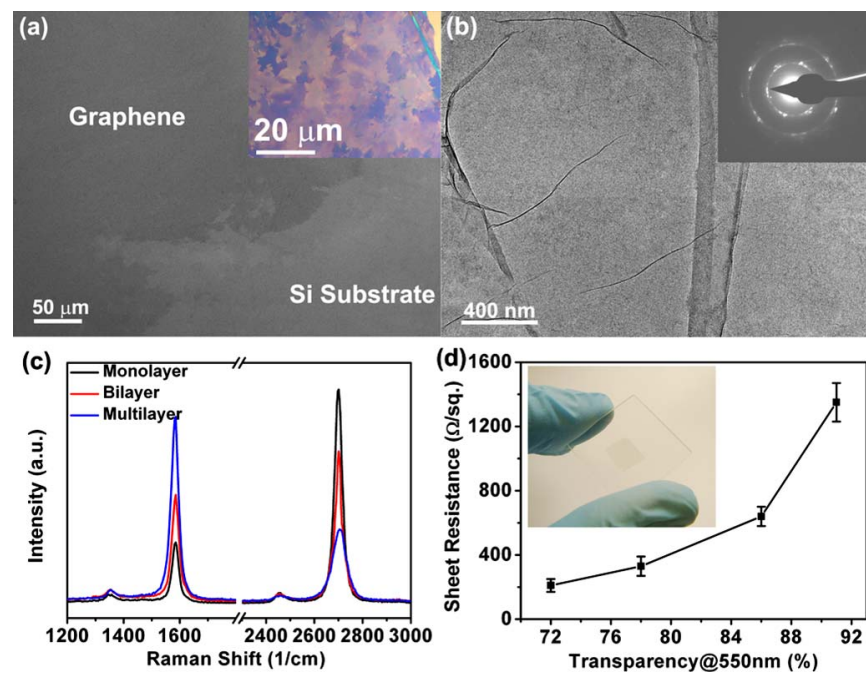

FIG. 1. (Color online) (a) SEM image of graphene film on Si substrate. (Inset: Optical microscopy image of the graphene film on $\mathrm{SiO}_{2} / \mathrm{Si}$ substrate.) (b) HRTEM image of graphene film in low magnification. Inset: Electron diffraction pattern from the unfolded region. (c) Raman spectra of graphene films of different layer thickness. (d) Sheet resistance vs transparency curves of graphene films. (Inset: Optical image of a graphene film on glass with $86 \%$ transparency.) 

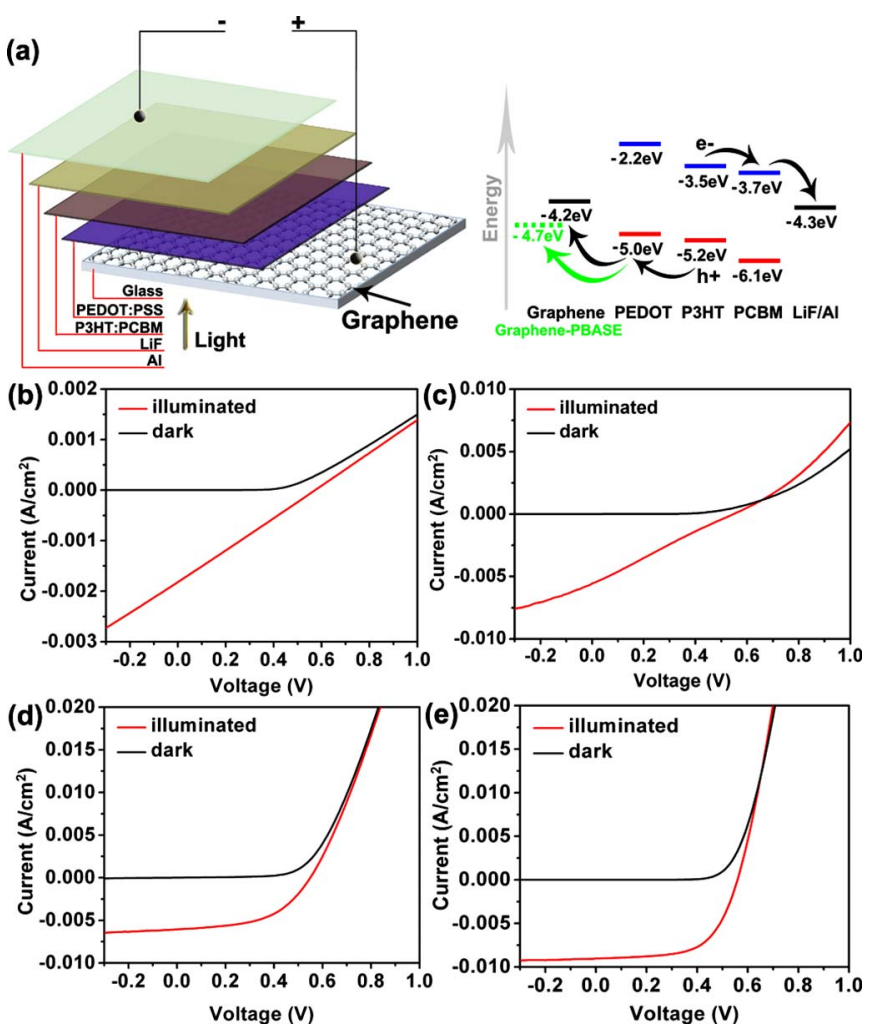

FIG. 2. (Color online) (a) Energy diagram of the fabricated device with structure graphene/PEDOT:PSS/P3HT:PCBM/LiF/Al. [(b)-(e)] Currentvoltage characteristics of the photovoltaic devices based on graphene films in dark and under illumination, where (b) is from pristine graphene film, (c) graphene film treated by UV light, (d) graphene film modified by PBASE, (e) ITO anode for comparison.

fects, which is evident of the highly crystalline and nearly defect-free graphene layers. For $6-30 \mathrm{~nm}$ thick graphene film, the average sheet resistance varies from 1350 to $210 \Omega /$ sq with an optical transparency from $91 \%$ to $72 \%$ in the visible light wavelength range [Fig. 1(d)]. These values are lower than the sheet resistance of chemically processed graphene oxide films by at least two to three orders of magnitude. The transparency and conductivity of CVD graphene is lower than that of ITO, which has sheet resistance of $10 \Omega /$ sq on glass at $80 \%$ transparency and $100-300 \Omega /$ sq on polyethylene terephthalate.

Figure 2(a) shows the device structure of the ITO-free solar cell using the graphene anode. Poly (3,4-ethylenedioxythiophene):poly (styrenesulfonate) (PEDOT:PSS) was spin coated and then annealed at $140{ }^{\circ} \mathrm{C}$ for $15 \mathrm{~min}$, producing a film of $40 \mathrm{~nm}$ thickness. The work function of graphene is $4.2 \mathrm{eV}$, while the highest occupied molecular orbital of poly(3-hexyl)thiophene (P3HT) is $5.2 \mathrm{eV}$. This indicates a large hole-injection barrier between graphene film and P3HT. The introduction of PEDOT facilitates the hole injection/ extraction as a buffer layer. ${ }^{16}$ The third layer is a bulkheterojunction composite of $\mathrm{P} 3 \mathrm{HT}$ and phenyl- $\mathrm{C}_{61}$-butyric acid methyl ester (PCBM), which provides the active layer $(160 \mathrm{~nm})$. Finally, $\mathrm{LiF}$ and aluminum were thermally evaporated to form the cathode, respectively. Figure 2(b) shows the current density-voltage characteristic of photovoltaic cells based on pristine graphene film using a simulated of AM1.5 G illumination $\left(P_{\mathrm{IN}}=100 \mathrm{~mW} / \mathrm{cm}^{2}\right)$ under nitrogen atmosphere. The photovoltaic cell had an open-circuit voltage $\left(V_{\text {oc }}\right)$ of $0.32 \mathrm{~V}$, a short-circuit current density $\left(J_{\mathrm{sc}}\right)$ of
TABLE I. Short-circuit current density $\left(J_{\mathrm{sc}}\right)$, open-circuit voltage $\left(V_{\mathrm{oc}}\right), \mathrm{FF}$ and PCE of various photovoltaic devices.

\begin{tabular}{lcccc}
\hline \hline \multicolumn{1}{c}{ Cell devices } & $\begin{array}{c}J_{\mathrm{sc}} \\
\left(\mathrm{mA} / \mathrm{cm}^{2}\right)\end{array}$ & $\begin{array}{c}V_{\mathrm{oc}} \\
(\mathrm{V})\end{array}$ & $\begin{array}{c}\mathrm{FF} \\
(\%)\end{array}$ & $\begin{array}{c}\text { PCE } \\
(\%)\end{array}$ \\
\hline Pristine graphene & 2.39 & 0.32 & 27 & 0.21 \\
Graphene treated by UV & 5.56 & 0.55 & 24.3 & 0.74 \\
Graphene modified by PBASE & 6.05 & 0.55 & 51.3 & 1.71 \\
ITO & 9.03 & 0.56 & 61.1 & 3.10 \\
\hline \hline
\end{tabular}

$2.39 \mathrm{~mA} / \mathrm{cm}^{2}$, and a fill factor (FF) of $27 \%$. The overall PCE for this device was therefore only $0.21 \%$. The poor performance was attributed to the hydrophobic property of graphene which makes uniform coating of PEDOT:PSS impossible. As explained, the PEDOT:PSS layer is essential to reduce the hole injection barrier on graphene and reduce the surface roughness via its planarization effect. ${ }^{17}$

To improve the surface wettability, the graphene anode was modified by UV/ozone treatment. It has been demonstrated that the hydrophobic polymer structures with aromatic rings can be turned into hydrophilic ones by the UV/ozone treatment because of the introduction of $\mathrm{OH}$ and $\mathrm{C}=\mathrm{O}$ groups ${ }^{18}{ }^{18}$ Indeed, after the UV treatment of graphene film for $10 \mathrm{~min}$, we can obtain an open-circuit voltage $\left(V_{\text {oc }}\right)$ of $0.55 \mathrm{~V}$, a short-circuit current density $\left(J_{\mathrm{sc}}\right)$ of $5.56 \mathrm{~mA} / \mathrm{cm}^{2}$, and a FF of $24.3 \%$ [Fig. 2(c)]. This increased the PCE to $0.74 \%$. Although the cell efficiency was improved, the reduction in FF has a negative effect on the device result. The decrease in FF reflects the increase in series resistance of the cell. In other words, although the surface has been rendered more hydrophilic by the presence of oxygenated groups, the good conducting properties of graphene has been compromised, due to the inevitable disruption of the aromatic structures by covalent bonding with oxygen groups. In this regard, we found that noncovalent functionalization with pyrene buanoic acid succidymidyl ester (PBASE) produces the best performance. Graphene anode modified by self-assembled PBASE exhibited excellent performance characteristics, as follow: $V_{\mathrm{oc}}=0.55 \mathrm{~V}, J_{\mathrm{sc}}$ $=6.05 \mathrm{~mA} / \mathrm{cm}^{2}, \mathrm{FF}=51.3 \%$, and $\mathrm{PCE}=1.71 \%$ [Fig. 2(d)]. The dramatic enhancement in device metrics with PBASE incorporation can be attributed to several factors. First, the increase in work function of graphene from 4.2 to $4.7 \mathrm{eV}$ after PBASE modification is beneficial in two aspects: (i) an increase in the open circuit potential for efficient electronhole separation and (ii) alignment of the Fermi level of graphene very close to the highest occupied molecular orbital of PEDOT:PSS for efficient hole collection. Second, overall high conductivity is maintained because noncovalent functionalization did not interrupt significantly the $\pi$-conjugation in graphene. Finally, PBASE improves the hydrophilic property of graphene and allows PEDOT:PSS to wet the surface effectively, which result in better planarization of the film surface and facilitation of hole injection. For the purpose of comparison, the photovoltaic performance of a control device made with ITO anode is shown in Fig. 2(e), where the device parameters are $V_{\mathrm{oc}}=0.56 \mathrm{~V}, \quad J_{\mathrm{sc}}$ $=9.03 \mathrm{~mA} / \mathrm{cm}^{2}, \mathrm{FF}=61.1 \%$, and $\mathrm{PCE}=3.10 \%$. This indicates that the PCE of the solar cells made with graphene anode has reached $55.2 \%$ of that of a structurally identical cell with ITO anode. Table I compares short-circuit current density $\left(J_{\mathrm{sc}}\right)$, open-circuit voltage $\left(V_{\mathrm{oc}}\right), \mathrm{FF}$, and PCE of or- 

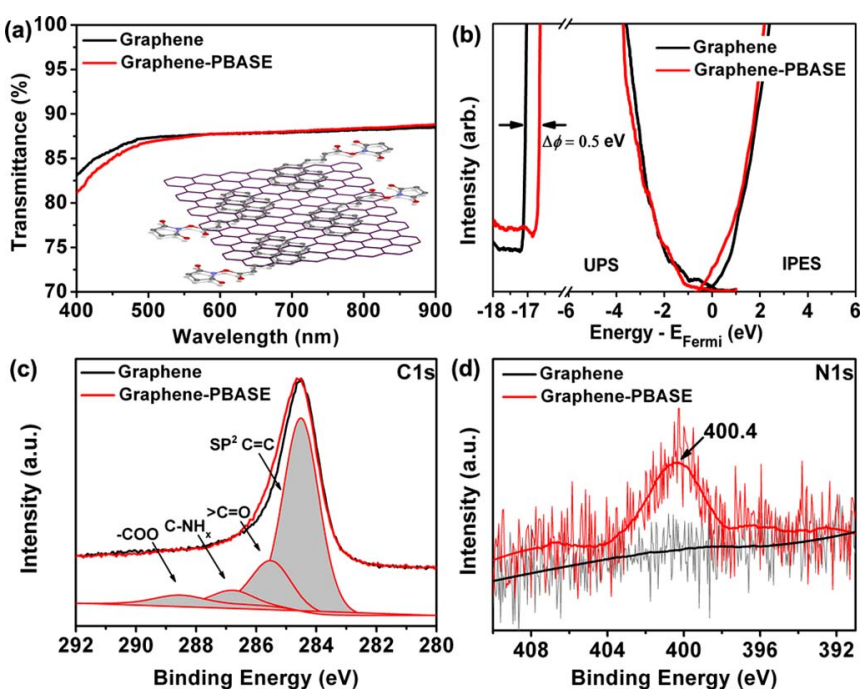

FIG. 3. (Color online) Graphene films before and after PBASE modification. (a) Transmittance spectra. Inset: A schematic diagram of the graphene modified by PBASE. (b) Combined UPS and IPES spectra. [(c) and (d)] XPS C $1 s$ and $\mathrm{N} 1 s$ core-level spectra, respectively.

ganic solar cells using graphene-based anodes modified by different surface treatments.

In the solution-processed of organic solar cells, the anode layer should possess good hydrophilicity to allow the spreading of PEDOT/PSS. In order to tune the surface wettability without degrading the conductivity, the graphene film was modified with a self-assembled molecular system: PBASE. The PBASE molecules stack on the surface of graphene sheet with a face-to-face orientation via $\pi$ - $\pi$ interactions [the inset of Fig. 3(a)]. In this case, graphene films on glass were incubated in $5 \mathrm{mM}$ of PBASE/ dimethylformaldehyde (DMF) solution in $80{ }^{\circ} \mathrm{C}$ for $10 \mathrm{~h}$. After that, the sample was rinsed 3 times in pure DMF to remove the free PBASE. We found that the noncovalent functionalization has negligible effect on the optical adsorption of graphene film in the visible region [Fig. 3(a)], this is expected because the optical gap of PBASE is in the UV region. Moreover, the changes in the electronic energy levels of graphene before and after modification with PBASE were investigated with ultraviolet photoelectron (UPS) as well as inverse photoemission spectroscopy (IPES). The spectra of UPS and IPES in Fig. 3(b) were aligned with respect to Fermi energy to show the valence band and conduction band edges. The surface transport gap can be judged from the gap region between the composite spectra. It can be seen that graphene, before or after modification with PBASE, maintains a nearly zero-gap condition. By measuring the difference between photoexcitation energy and spectral width, given by $\phi=h \nu-W$, the work functions $(\phi)$ of graphene and graphene modified with PBASE were determined to be 4.2 and $4.7 \mathrm{eV}$, respectively, where $h \nu$ is UV light energy (He I, $21.2 \mathrm{eV}$ ) and $W$ is the energy difference between the Fermi level $\left(E_{F}\right)$ and secondary electron cut-off. The adsorption of PBASE on the surface of the graphene film was also verified by x-ray photoelectron spectroscopic (XPS) measurements. In the $\mathrm{C} 1 s$ core spectra [Fig. 3(c)], both samples exhibit a large peak at around $284.8 \mathrm{eV}$, which can be attributed to the $s p^{2}$ carbon atoms of the carbon skeleton. However, the C $1 s$ peak of graphene-PBASE was slightly broadened asymmetrically after the noncovalent functionalizaion. The peak at $286.8 \mathrm{eV}$ arises from $\mathrm{C}-\mathrm{N}$, in agreement with the $\mathrm{N} 1 s$ spectrum explained later. The shoulder in the main peak can be split into another two smaller peaks, with binding energies of 285.5 and $288.7 \mathrm{eV}$, and can be assigned $\mathrm{C}=\mathrm{O}$ and COOspecies from PBASE, respectively. ${ }^{19}$ For $\mathrm{N} 1 s$ core spectra, the graphene film exhibits a well-defined peak at $400.4 \mathrm{eV}$ [Fig. 3(d)] attributable to the presence of pyrrolelike nitrogen atom (N $1 s)$ in PBASE. ${ }^{20}$

In summary, large-area, continuous graphene films with high optical transparency and electric conductivity can be produced by CVD method. As an anode in organic photovoltaic devices, graphene is very promising to replace ITO due to its inherent low cost fabrication process and the outstanding conductivity and transparency properties. The main drawback of graphene film is its poor hydrophilicity, leading to a negative effect on the construction of solution-processed devices, but the results of our work demonstrate that this can be overcome by modifying its surface using noncovalent chemical functionalization method. Considering the mechanical strength and flexibility of graphene and its superior conductance properties, it can be envisaged that new applications in plastic electronics and optoelectronics will soon emerge using this new class of CVD graphene materials.

This work is supported by the NRF-CRP award "Graphene and Related Materials and Devices" (Grant No. R-143-000-360-281). We are grateful to Professor B. H. Hong for providing technical advice for the CVD growth of graphene films.

${ }^{1}$ A. K. Geim and K. S. Novoselov, Nature Mater. 6, 183 (2007).

${ }^{2}$ K. S. Novoselov, A. K. Geim, S. V. Morozov, D. Jiang, Y. Zhang, S. V. Dubonos, I. V. Grigorieva, and A. A. Firsov, Science 306, 666 (2004).

${ }^{3}$ A. Rycerz, K. J. Tworzydlo, and C. W. J. Beenakker, Nat. Phys. 3, 172 (2007).

${ }^{4}$ Z. C. Wu, Z. H. Chen, X. Du, J. M. Logan, J. Sippel, M. Nikolou, K. Kamaras, J. R. Reynolds, D. B. Tanner, A. F. Hebard, and A. G. Rinzler, Science 305, 1273 (2004).

${ }^{5}$ M. Zhang, S. L. Fang, A. A. Zakhidov, S. B. Lee, A. E. Aliev, C. D. Williams, K. R. Athinson, and R. H. Baughman, Science 309, 1215 (2005).

${ }^{6}$ S. I. Na, S. S. Kim, J. Jo, and D. Y. M. Kim, Adv. Mater. 20, 4061 (2008)

${ }^{7}$ J. F. Li, L. B. Hu, L. Wang, Y. X. Zhou, G. Grüner, and T. J. Marks, Nano Lett. 6, 2472 (2006).

${ }^{8}$ H. S. Peng, J. Am. Chem. Soc. 130, 42 (2008).

${ }^{9}$ X. Wang, L. J. Zhi, and K. Y. Müllen, Nano Lett. 8, 323 (2008).

${ }^{10}$ J. B. Wu, H. A. Becerril, Z. N. Bao, Z. F. Liu, Y. S. Chen, and P. Peumans, Appl. Phys. Lett. 92, 263302 (2008).

${ }^{11}$ C. Gomez-Navarro, R. T. Weitz, A. M. Bittner, M. Scolari, A. Mews, M. Burghard, and K. Kern, Nano Lett. 7, 3499 (2007).

${ }^{12}$ K. S. Kim, Y. Zhao, H. Jang, S. Y. Lee, J. M. Kim, K. S. Kim, J.-H. Ahn, P. Kim, J.-Y. Choi, and B. H. Hong, Nature (London) 457, 706 (2009).

${ }^{13}$ S. Roddaro, P. V. Piazza, V. Pellegrini, and F. Beltram, Nano Lett. 7, 2707 (2007).

${ }^{14}$ J. H. Warner, M. H. Rümmeli, T. Gemming, B. Büchner, and G. A. D. Briggs, Nano Lett. 9, 102 (2009).

${ }^{15}$ L. G. Cancado, A. Reina, J. Kong, and M. S. Dresselhaus, Phys. Rev. B 77, 245408 (2008).

${ }^{16}$ S. Günes, H. Neugebauer, and N. S. Sariciftci, Chem. Rev. (Washington, D.C.) 2007, 1324

${ }^{17}$ M. C. Scharber, D. Mühlbacher, M. Koppe, P. Denk, C. Waldauf, A. J. Heeger, and C. J. Brabec, Adv. Mater. 18, 789 (2006).

${ }^{18}$ T. N. Murakami, Y. Fukushima, Y. Hirano, Y. Tokuoka, M. Takahashi, and N. Kawashima, Colloids Surf., B 29, 171 (2003).

${ }^{19}$ N. I. Kovtyukhova, T. E. Mallouk, L. Pan, and E. C. Dickey, J. Am. Chem. Soc. 125, 9761 (2003).

${ }^{20}$ T. I. T. Okpalugo, P. Papakonstantinou, H. Murphy, J. McLaughlin, and N. M. D. Brown, Carbon 43, 153 (2005). 